Présence de cristaux sur les biopsies de greffons rénaux : fréquence, étiologie et pronostic

A. Garstka ${ }^{1}$, M. Haeck ${ }^{1}$, V. Gnemmi ${ }^{1}$, A. Lionet ${ }^{1}$, M. Frimat ${ }^{1}$, D. Bazin ${ }^{2}$, J.P. Haymann ${ }^{3}$, M. Daudon ${ }^{3}$, C. Noël ${ }^{1}$

1 Néphrologie, Hôpital Huriez, Lille, France

2 Chimie de la Matière Condensée de Paris, Collège de France, Paris, France

3 Service d'Explorations Fonctionnelles, Hôpital Tenon, Paris, France

Adresse e-mail : antoine.garstka@ chru-lille.fr (A. Garstka)

\title{
Introduction
}

Bien que la présence de cristaux tubulo-interstitiels au sein de biopsies de greffon rénaux soit décrite, leur signification reste imprécise [1,2]. L'objectif de ce travail rétrospectif et monocentrique était donc de définir leur fréquence, leur étiologie et leur impact pronostique.

Patients et méthodes Entre 1996 et 2012, 1621 biopsies ont été réalisées chez 1040 greffés rénaux. Des cristaux ont étés objectivés sur 130 biopsies $(8,1 \%)$ chez 78 patients $(7,5 \%)$, et avant 2 ans de greffe dans $84,5 \%$ des cas. Les paramètres clinico-biologiques de 55 patients porteurs de cristaux $(\mathrm{Pcx}+)$ ont été comparés à un groupe témoin de 100 patients greffés $\left(\mathrm{Pcx}^{-}\right)$.

Résultats Dans le groupe Pcx+, nous avons constaté significativement plus de lésions vasculaires ( $49 \%$ vs $25,1 \%, \mathrm{p}<0,001$ ) et de microvacuolisation tubulaire (58\% vs 37,1\%, p =0,004). Nous n'avons pas constaté de différence entre les deux groupes pour les caractéristiques des donneurs, des receveurs, leurs traitements ou les facteurs d'agression du greffon. Parmi les paramètres étudiés à $\mathrm{j} 15, \mathrm{~m} 3, \mathrm{~m} 6, \mathrm{~m} 12$ et $\mathrm{m} 24$, l'hyperparathyroïdie (HPT) durant la première année de greffe était significativement plus marquée dans le groupe Pcx+ vs Pcx- : J15:278 vs $160 \mathrm{pg} / \mathrm{mL}(\mathrm{p}=0,001)$; m3 : 211 vs $123 \mathrm{pg} / \mathrm{mL}(\mathrm{p}=0,003) ; \mathrm{m} 6: 193$ vs $105 \mathrm{pg} / \mathrm{mL}(\mathrm{p}=0,001) ; \mathrm{m} 12: 198$ vs $117 \mathrm{pg} / \mathrm{mL}$ $(\mathrm{p}=0,005)$. Une PTH supérieure au 75e percentile était associée à un risque relatif de calcifications sur la biopsie de 4,2 à j15 (IC 1,9-9,17, p < 0,001), de 3,88 à m3 (IC 1,79-8,43; p < 0,001) et de 3,04 à $\mathrm{m} 6$ (IC 1,2-7,6; $\mathrm{p}=0,016$ ). La calciurie n'était ni corrélée à la survenue de cristaux ni au niveau de PTH. Le débit de filtration glomérulaire estimé à deux ans de greffe n'était pas statistiquement différent entre les deux groupes.

\section{Discussion et conclusion}

Au total, la découverte de cristaux tubulo-interstitiels est fréquente dans les deux premières années de greffe et associée à une HPT initiale plus intense. Si le DFG à 2 ans de greffe est comparable, les conséquences à long terme restent à déterminer.

\section{Déclaration d'intérêts}

Les auteurs n'ont pas transmis de déclaration de conflits d'intérêts.

Références

[1] Gwinner W, et al. Am J Transplant 2005;5:1934-41.

[2] Bagnasco S, et al. Nephrol Dial Transplant 2009;24:1319-25. 